This is a self-archived version of an original article. This version may differ from the original in pagination and typographic details.

Author(s): Tomkins, Joseph; Kotiaho, Janne Sakari; LeBas, Natasha

Title: Major difference in minor allometries: a reply to Moczek

Year: 2006

Version: Published version

Copyright: (c) 2006 by The University of Chicago.

Rights: In Copyright

Rights url: http://rightsstatements.org/page/InC/1.0/?language=en

Please cite the original version:

Tomkins, J., Kotiaho, J. S., \& LeBas, N. (2006). Major difference in minor allometries: a reply to Moczek. The American Naturalist, 167, 612-618. https://doi.org/10.1086/501077 
VOL. 167 , NO. 4 THE AMERICAN NATURALIST APRIL 2006

\title{
Major Differences in Minor Allometries: A Reply to Moczek
}

\author{
Joseph L. Tomkins, ${ }^{1, *}$ Janne S. Kotiaho, ${ }^{2, \dagger}$ and Natasha R. LeBas,
}

1. Centre for Ecology and Conservation, University of Exeter in Cornwall, Tremough, Penryn TR10 9EZ, United Kingdom; 2. Department of Biological and Environmental Science, University of Jyväskylä, P.O. Box 35, 40014 Jyväskylä, Finland; 3. School of Animal Biology, University of Western Australia, Nedlands, Western Australia, 6009 Australia

Submitted May 9, 2005; Accepted December 14, 2005; Electronically published March 13, 2006

ABSTRACT: By comparing alternative measurements of horn length in the beetle Onthophagus taurus, we have investigated why scaling patterns differ between laboratories. We show that some measurements are confounded by including part of the head in the horn size measurement and consistently underestimate the allometry of horns in minor males. Our data show how linear measures that avoid confounding horn length with head size produce scaling patterns that support a positive allometry rather than a reprogramming model of horn growth. We also found horn volume was highly positively allometric this estimate of growth therefore further supports a positive allometry model.

Keywords: allometry, reprogramming, Onthophagus taurus, development, alternative tactics.

We recently suggested that patterns of dimorphic allometry in the horns of some beetles were more readily explained by a reaction norm of extreme positive allometry followed by a constraint (Tomkins et al. 2005). This is in contrast to existing models, which rely on developmental reprogramming at a body size threshold (Emlen and Nijhout 1999, 2000, 2001; Nijhout 1999, 2003; Emlen 2000; Moczek and Nijhout 2002). Moczek (2006, in this issue) has suggested that some of the congruence between our positive-allometry model and the data we presented on horn allometries arises from the method we used to measure the beetles' horns. Our method does indeed differ

\footnotetext{
* E-mail: j.l.tomkins@ex.ac.uk.

† E-mail: janne.kotiaho@bytl.jyu.fi.

¥ E-mail: nlebas@cyllene.uwa.edu.au.
}

Am. Nat. 2006. Vol. 167, pp. 612-618. (c) 2006 by The University of Chicago. 0003-0147/2006/16704-41063\$15.00. All rights reserved. from those used by other labs, and Moczek is correct to ask how the differences have influenced the data and the conclusions drawn.

To summarize Moczek's article, there are two substantive points. The first point is that landmarks affect the data in morphometric studies, and the second is that the biology should drive the methodology used and the landmarks chosen. These are important points, and they culminate in the impression that the fit of the data to our model might have been due to our measure of horn size. Here we deal with each point in turn and reassess the fit of several different measures of horn length to our model.

\section{Straight Lines versus Curved Lines}

Moczek (2006, in this issue) is correct that we have favored the linear measure of horn "height" over what he describes as the horn "length" measurement used by labs in the United States. Moczek shows that these different methods yield different allometric slopes and, in particular, that the horn allometry in minor males is much less steep when using the length method than when using the height method. This is an important finding, because it makes log horn-on-log pronotum width allometries that are derived from the length measurement more sigmoid and therefore more consistent with the reprogramming model. With respect to our own data, we also acknowledge that there are limitations to the height measurement we used, in particular because it did not measure the curve of the horns in major males. This results principally in the length measurement having a higher asymptote (in majors) than horn height measurements. Hence, we agree with Moczek; it does matter which measurement is used in the analysis of static allometries. Furthermore, those measurements that reflect the changing shapes of the structures in question are preferable.

Moczek (2006, in this issue) found, using the length measurement, that the horn allometry of minor males in his sample was 1.98; this is an unusually steep allometric slope. For example, the slopes of numerous morphological traits, such as elytra and wings, in Onthophagus taurus males vary only between 0.76 and 1.11 (Tomkins et al. 2005). So Moczek's data, despite being measured differ- 

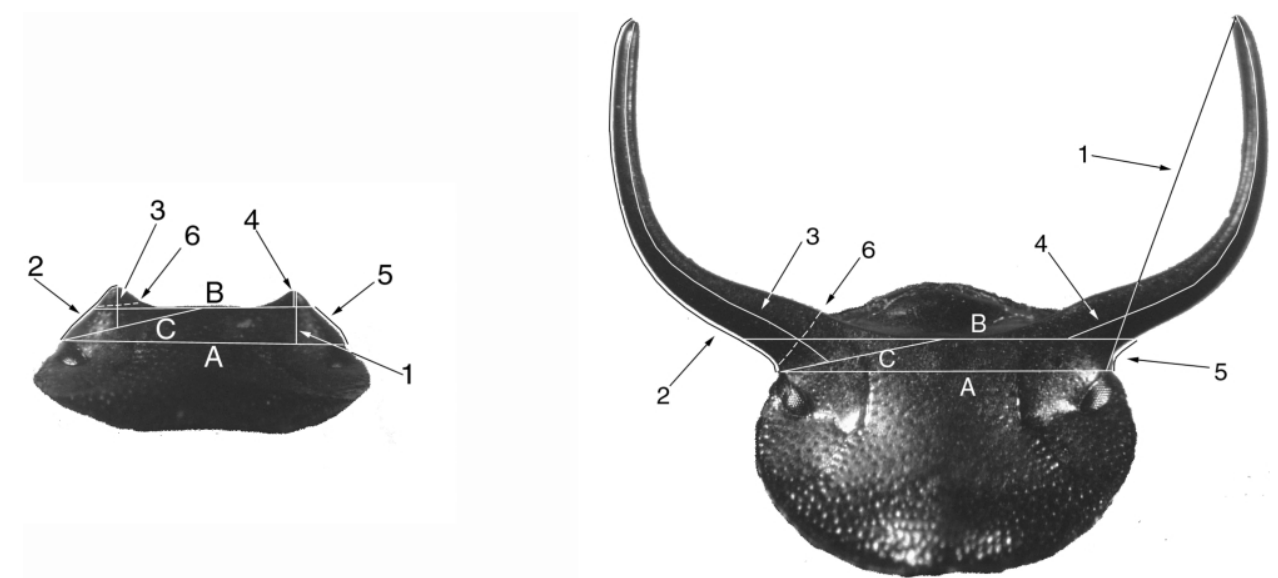

Figure 1: Anterior view of the head of a minor (left) and a major (right) male Onthophagus taurus, showing landmark lines $A, B$, and $C$ and measurements 1-6.

ently, do show horn growth in minor males occurring with an exponent greater than that for other morphological traits. This finding is itself incompatible with models that assume no or only "rudimentary" horn growth in minor males (Emlen and Nijhout 1999, 2000, 2001; Nijhout 1999, 2003; Emlen 2000; Moczek and Nijhout 2002). Finally, it is peculiar that the difference in the measurement techniques made a dramatic difference to the slope of horn length in the straight-horned minor males but changed the major horn slope comparatively little. Below we show why this occurs.

\section{Landmark Choice and Allometry}

Moczek (2006, in this issue) makes the point that landmark choice should be driven by the biological question at hand, something with which we can only agree. Here we extend Moczek's comparison of measurements to analyze a range of horn length measures and landmarks in an attempt to understand why the alternative measures diverge as they do. Moczek has shown that in majors, the horn curve is an important character to include in the measure of horn length; here we concentrate on why the allometries of horns in minor males are so different, even though these horns are straight.

In the sample presented in Tomkins et al. (2005), we used the height of the horn above the top of the head in minor males. In majors, because the horn begins to grow out from the side of the head, we used the top of the eye. The shift in landmark is less than ideal, but it was used to accommodate the change in the angle of the horns in relation to the head as they grow; our results below suggest that there was little error generated by this method, in terms of the conclusions we drew. Moczek's interpretation of our height measure is slightly different from the one we actually used, because in both minors and majors, the corner of the eye is used by Moczek and the height is the distance to level with the horn tip, whereas we measured directly to the horn tip. This is a misunderstanding of our making: we should have been clear about the landmarks used in our article. This difference in landmark does not change Moczek's important point that the length measure may be superior because it incorporates changes in shape and is therefore a better reflection of growth of the horn. Here we compare the following measures: the measure of height used by Moczek but directly to the horn tip, Moczek's length measure, an improvement on our original height measurement that uses a single basal landmark at the top of the head but follows the horn's curve, and finally, in an attempt to account for the cone shape of the horn, an important consideration suggested by Moczek, an estimate of the horn volume.

We measured a sample of Onthophagus taurus from Western Australia. Pronotum width was measured to within $0.01 \mathrm{~mm}$ with Mitotoyo digital calipers. The beetle's head was detached and mounted on a pin under an Olympus SZH dissecting microscope, and images were captured on an Olympus DPII digital camera and analyzed using Scion Image. On each image, a line was drawn to locate the top of the eyes (line $A$ ), a line parallel to this was drawn to locate the lowest point of the top of the head between the horns (line $B$ ), and finally, a line was drawn from the center of the top of the head to the top of the right eye (line $C$ ). These three lines provided additional landmarks for the measurements. Line $B$ was used as a landmark to measure horn growth above the extended contour of the head. Line $C$ was used as a landmark to 
measure horn growth from a diagonal that could approximate the base of the horn.

We took six measurements (fig. 1): (1) a "height" measure from the top of the eye with a straight line to the horn tip, similar to Moczek's example; (2) Moczek and others' favored length measure; (3) a measure from the middle of the horn tip, following the horn curve to the line $C$, the diagonal between the center of the head and the top of the eye; (4) a measure from the middle of the horn tip, following the horn curve to line $B$, the horizontal line level with the lowest point at the top of the head; (5) the distance from the top of the eye to line $B$; and (6) the width of the base of the horn perpendicular to its long axis. Measurements 1-4 were designed to vary the proportion of the head that was incorporated in the measurement of the horn. Measurement 5 was taken to make it possible to show an example of how much of the "length" measurement was confounded by a measure of the size of the head. Measurement 6 allowed us to estimate the volume of the horn.

Our four linear measures of the horn all produced the characteristic sigmoid pattern of horn length on pronotum width (fig. $2 A$ ). In minor males, the measures differ clearly in elevation; in major males, the pattern is less clear, albeit confirming that the height measure does yield shorter horn lengths in majors, as expected, because it does not account for the curve in the major horn, as Moczek showed. When the measurements are log-transformed (fig. $2 B$ ), the results similarly support Moczek's finding that the height measure has a steeper allometry (all results are expressed as reduced major axis slopes and 95\% confidence intervals; 5.19, 8.224.57) than the length measure $(3.18,3.69-2.75)$; they also show that our additional measure $3(7.37,8.22-6.52)$ and measure $4(13.1,14.5-11.8)$ reveal even steeper allometries in minor males than the other measurements. The horns of minor males are straight; so although we used a linear measure (measure 1) in Tomkins et al. (2005), method 4, where the curve is followed, improves on the previous method only in the measurement of majors' horns. Measure 4 has an allometry for minors very similar to the one we reported originally $(12.2,14.4-10.4$; Tomkins et al. 2005).

As pointed out by Moczek, useful measures of horn length take account of the cone shape and curve of the horn; importantly, however, they should also not be confounded by other traits. This appears to be particularly important when the trait of interest is very small. Figure 1 shows how in small minors the horn length measure can be twice as long as the horn height measure Moczek used and many times longer than our measure 4 . This is because the length measure traces the outline of the head and horn rather than measuring the horn itself. The effect of measuring the head as part of horn length can be seen in figure $2 C$, where $\log$ (horn length) and $\log$ (horn length minus measurement 5) are plotted against log pronotum width. Evidently, the length measure is confounded in small males by the inclusion of a part of the head into the measure. In small minor males, the part of the head that is being included forms as much as $90 \%$ of the total "horn length" and thus has an extreme effect on the allometry of horn length. Figure $2 D$ shows the contribution of measurement 5 (head height) to horn length; in minor males, most of the horn length measure is a measure of head height, but the contribution is negligible in majors. Measurements of horn length using the length method will always have an allometry in minor males that reflects head size rather than horn length. Our measure, by using the top of the head in minors, avoided inclusion of the head in our measurements of the horn, thus revealing the allometry of only the horns.

Where landmarks have been used that include a proportion of some other body part, the slope will always be affected. Where the trait of interest has a very steep allometry, the smallest individuals necessarily have relatively tiny traits compared to large individuals, and therefore the confounding effect will always increase in smaller individuals and (where the allometry is positive) will result in an underestimate of the true allometry. This is likely to confound the interpretation of allometric patterns in other species unless the landmarks measure only the trait of interest and do not include other measures of body size. In the Onthophagus binodis sample in Tomkins et al. (2005), the landmarks were chosen so that only the horn was measured, contributing to the good fit of these data to the model. Finding reliable landmarks for the measurement of the horn and no other tissue is not easy, and no doubt improvements can be made to our measurement 4 ; in particular, these measures still change slightly in ma-

Figure 2: $A$, Linear scale relationship of horn length on pronotum width for the four measures of horn length and $B$, the same data after the log transformation. Gray circles $=$ height measure 1; squares $=$ length measure 2; open circles $=$ measure 3; filled circles $=$ modified height measure 4 . $C$, Log-transformed horn length plotted against log-transformed body size. Open circles show horn length measure 2, and filled circles show measure 2 after the distance from the top of the eye to the base of the horn (measure 5) has been subtracted. $D$, The change in contribution to horn length measure 2 of the distance from the top of the eye to the base of the horn (measure 5). E, Positive allometry of horn length, measured using the modified height measure 4. F, Positive allometry of horn volume: log horn volume, calculated from the formula for the volume of a cone, plotted against the estimate of the volume of the beetle. 

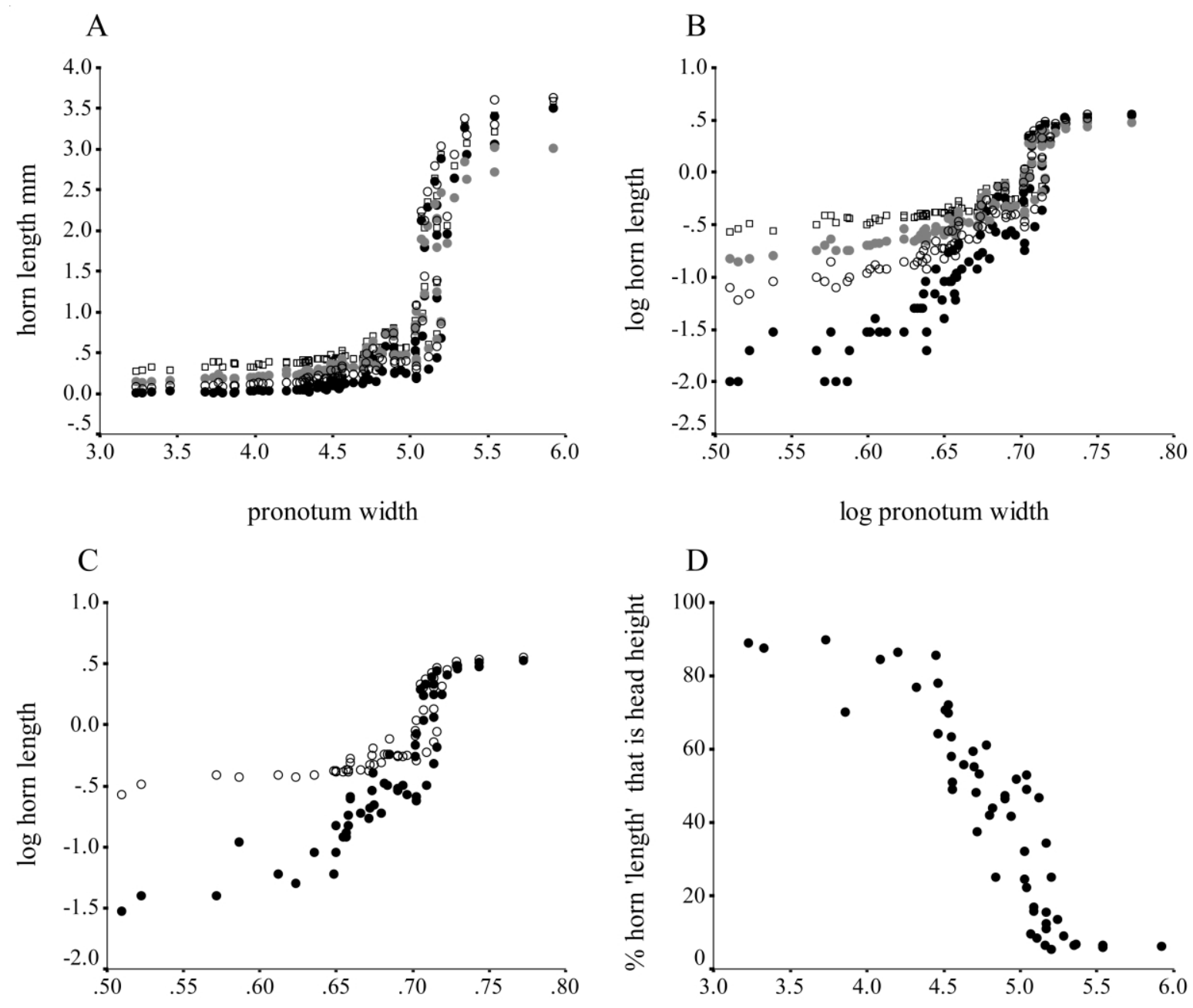

$\log$ pronotum width

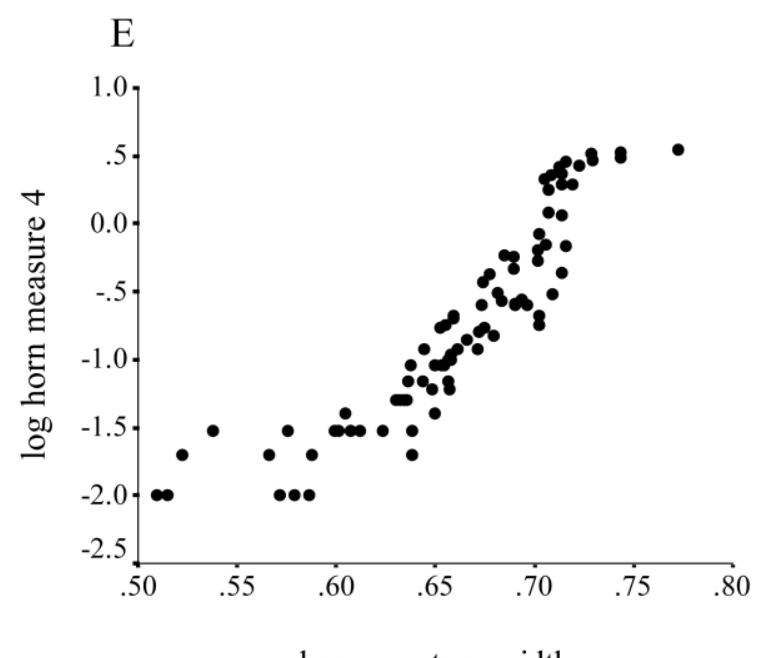

$\log$ pronotum width

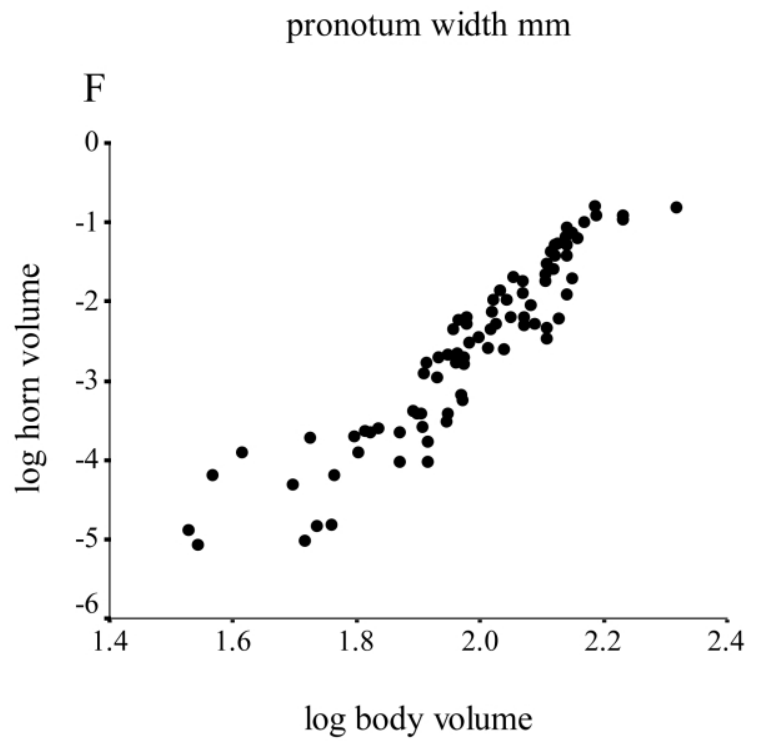


jors because the angle of the horn in relation to the head changes. Furthermore, the very smallest males had horns that were difficult to measure using measure 4 , causing the apparent flattening of the curve in those males. This is expected under the positive-allometry hypothesis because we expect the intercept of the allometry to be extremely small and therefore the horns in the smallest males to be very difficult to measure. The horns of the smallest males were $0.01 \mathrm{~mm}$ long, which is also at the limit of our measurement ability. When the trait is so small, measurement error in relation to trait size is likely to be a large factor, with small horns all being assigned the same, smallest unit of measurement. Because of the limited ability to measure a tiny amount of growth, the flattening of curves in the smallest males, even where only horn is measured, is likely to be a common feature of these types of allometries in this and other species.

We agree with Moczek that an approach that achieves a three-dimensional quantification of the horn size and shape, thus allowing accurate estimation of horn volume, would be a worthwhile advance. The possibility of using geometric morphometrics to measure the three-dimensional changes in horns does represent a real possibility for quantifying horn growth. The smooth contours of the horn provide few landmarks, however, and the changing angles of the horn and head will make this a technically difficult propositionbut it is something we look forward to seeing done. Such data would address Moczek's concern that simple linear measures do not capture the growth that is taking place in the cone-shaped horns of minors and the more tubular horns of the majors. Coming as close as we can to achieving this goal, we have estimated the volume of the horns based on the height from measure 4 and the width at the base of the horn perpendicular to the horn's long axis, measure 6 . We used these measures in the formula for the volume of a cone $\left([1 / 3] \pi r^{2} h\right)$ to estimate the volume of the horn; while not a perfectly accurate measure of horn volume, this calculation does give a good indication of the pattern of changes in horn volume. We assumed the horns of majors to be cones as well, but the error that this induces is of little concern, because the pattern in minors is of importance here and the greatest contribution to the variance in volume in major horns lies in the length component. When the volume of a cylinder rather than that of a cone is used, the results are qualitatively unchanged. The allometry of log horn volume on log body volume $\left(\right.$ pronotum $w^{2} \mathrm{dth}^{3}$ ) yields a pattern very similar to those we revealed in our original article, and here, with measure 4 (fig. 2E) showing a steep allometric slope for horn volume of minors $(6.4,5.7-7.13)$, that continues across all males up to an asymptote (fig. $2 F$ ). The relation of $\log$ horn length to log pronotum width need not be linear to support the positive-allometry model (Nijhout and
Wheeler 1996; fig. 3C); rather, the key is that there should not be a discontinuity and that there should be evidence for exponential growth in minor males (Tomkins et al. 2005). Hence, these results for horn volume are in line with the conclusion that horn growth in these beetles is a continuous reaction norm rather than a polyphenism (see also Nijhout 2003; Tomkins et al. 2005). Hence, we remain confident that any direct geometric morphometric measurements of horn volume will support our original model (Tomkins et al. 2005).

\section{Scale Matters}

Previously, we suggested (Tomkins et al. 2005) that earlier work had interpreted horn allometry in dung beetles incorrectly by failing to use the log scale. However, the problem of scaling has an additional facet; in allometric plots, the axis dimensions also affect the interpretation of curvilinear allometries. For example, statistical packages draw scatter plot axes with a ratio of $X$ to $Y$ such that traits that scale with isometry rarely have a slope of $45^{\circ}$. Moczek's (2006, in this issue) figure 2 is an example in which the range of the $Y$-axis is approximately 9.5 times that of the $X$-axis; nevertheless, the $X$-axis is drawn longer (also true of our fig. 2). The effect is to compress the $Y$-axis, foreshortening any nonlinearities. The benefit is that the nonlinearities are easier to distinguish; however, the cost is that the magnitude of the nonlinearities is magnified and that steep allometric slopes rarely appear so. We have plotted the horn allometries that are produced from our measurement 4 (fig. $3 A$ ) and Moczek's length measurement (fig. 3B) alongside Nijhout and Wheeler's (1996) prediction for a disk allometry without reprogramming (fig. 3C). Comparing figure $2 B$ and Moczek's (2006, in this issue) figure 1 with figure $3 A$ and $3 B$ reveals how axis scaling could affect the conclusions drawn from purely visual interpretation of the nonlinearities in the allometry. Where $\Delta X=\Delta Y$ (fig. $3 A, 3 B$ ), the reaction norm of horn length reveals the positive allometry across all males, even using Moczek's length measure (fig. 3B). Plots in which $\Delta X=\Delta Y$ provide a nonarbitrary scale by which divergent allometries can be compared graphically.

\section{Populations}

We disagree with Moczek's (2006, in this issue) argument that we generated a "discrepancy" by using populations other than one from the United States; after all, the utility of a model comes down to its generality. The populations from the United States and Australia do differ in the proportion of majors, and indeed, the reprogramming model was drawn up by workers using the U.S. population. However, in the same way that the reprogramming model was 
A

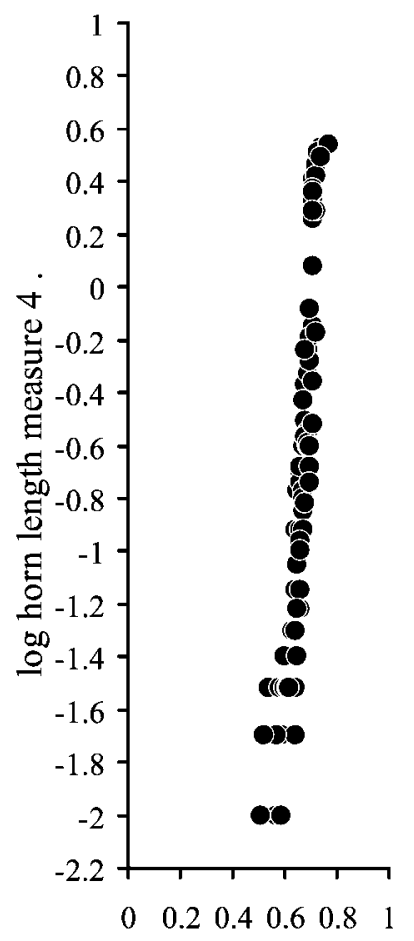

$\log$ pronotum width
B

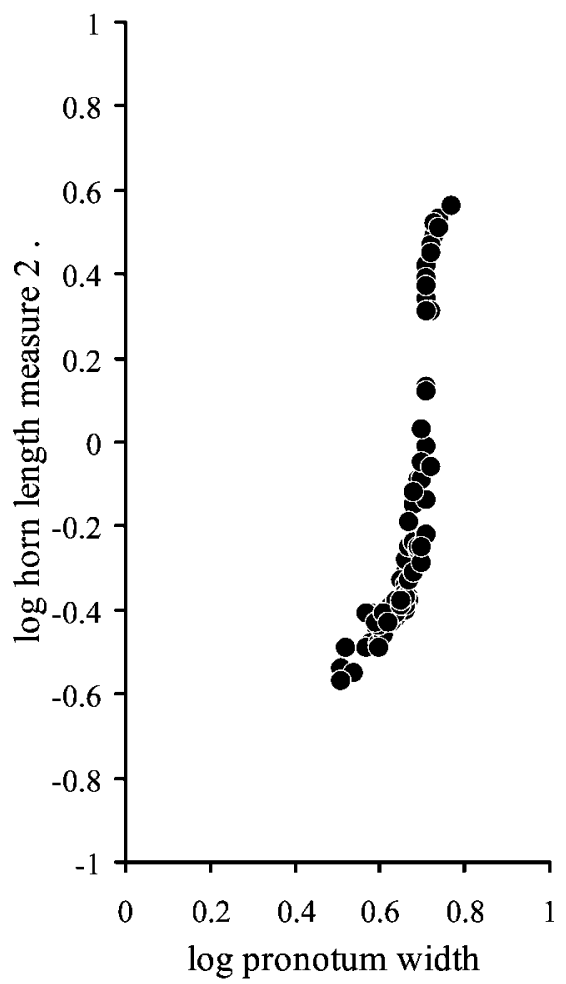

$\mathrm{C}$

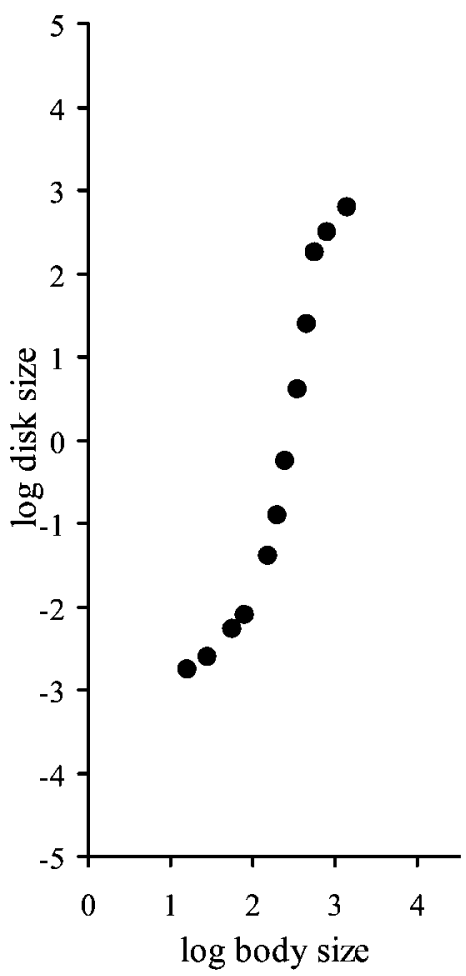

Figure 3: Horn length allometries drawn so that $\Delta X=\Delta Y$, thereby providing a nonarbitrary image of the scaling pattern. $A$, Modified height measure 4; $B$, Moczek's length measure; $C$, allometry of a nonreprogrammed disk, redrawn from Nijhout and Wheeler (1996).

not conceived as relevant only to U.S. populations of Onthophagus taurus, we do not feel that our model is specific to Western Australian populations of the beetle. Our model (Tomkins et al. 2005) explains how differences between populations like those in the United States and Australia can arise through changes in the intercept or slope of the horn allometry, and hence we do not feel that the model is at all confined to the Western Australian population. Indeed, we are confident that the model applies across numerous dimorphic taxa beyond onthophagine beetles, let alone to differences between $O$. taurus populations.

\section{Conclusion}

The data we have presented, measuring the horns in a number of different ways, show that the closer the measure comes to measuring only horn growth, the steeper and straighter the allometry in minor males becomes. The smooth transition from the head to the horn no doubt generated the ambiguity over where the horn starts and has caused the divergent results between labs. In terms of selecting appropriate landmarks, it is apparent that positive allometries are particularly sensitive to the inclusion of parts of other traits, where landmarks delineating the trait of interest are ambiguous. With respect to the conclusions drawn in our previous study, our results suggest that we were measuring meaningful changes in horn growth in minor males but underestimating the asymptote in majors. The length measure of Moczek and Emlen, in contrast, measures the asymptote in majors appropriately but confounds the length and allometry of horns of minors by inclusion of part of the head. In our survey of the horn length measures available with two-dimensional morphometrics, we found measurement 4 , and in particular the volume estimation, to reduce the amount of head size in the measurement of the horns and to therefore provide a better estimate of the true horn allometry and the rates of growth of these structures. Our new data hopefully provide convincing support for the hypothesis that horns 
grow extremely rapidly from the smallest minor male beetles up to the point where large males exhaust their resources for growth (Tomkins et al. 2005; cf. Knell et al. 2004).

\section{Acknowledgments}

J.L.T. is supported by a Royal Society University Research Fellowship, J.S.K. by the Academy of Finland, and N.R.L. by an Australian Research Council Fellowship. We thank L. Simmons for providing the beetles.

\section{Literature Cited}

Emlen, D. J. 2000. Integrating development with evolution: a case study with beetle horns. BioScience 50:403-418.

Emlen, D. J., and H. F. Nijhout. 1999. Hormonal control of male horn length dimorphism in the dung beetle Onthophagus taurus (Coleoptera: Scarabaeidae). Journal of Insect Physiology 45:45-53.

- 2000. The development and evolution of exaggerated morphologies in insects. Annual Review of Entomology 45:611-708.

. 2001. Hormonal control of male horn length dimorphism in Onthophagus taurus (Coleoptera: Scarabaeidae): a second critical period of sensitivity to juvenile hormone. Journal of Insect Physiology 47:1045-1054.

Knell, R. K., J. C. Pomfret, and J. L. Tomkins. 2004. The limits of elaboration: curved allometries reveal the constraints on mandible size in stag beetles. Proceedings of the Royal Society of London B 271:523-528.

Moczek, A. P. 2006. A matter of measurements: challenges and approaches in the comparative analysis of static allometries. American Naturalist 167:606-611.

Moczek, A. P., and H. F. Nijhout. 2002. Developmental mechanisms of threshold evolution in a polyphenic beetle. Evolution and Development 4:252-264.

Nijhout, H. F. 1999. Control mechanisms of polyphenic development in insects. BioScience 49:181-192.

2003. Development and evolution of adaptive polyphenisms. Evolution and Development 5:9-18.

Nijhout, H. F., and D. E. Wheeler. 1996. Growth models of complex allometries in holometabolous insects. American Naturalist 148: 40-56.

Tomkins, J. L., J. S. Kotiaho, and N. R. LeBas. 2005. Matters of scale: positive allometry and the evolution of male dimorphisms. American Naturalist 165:389-402.

Associate Editor: Troy Day Editor: Jonathan B. Losos 